\title{
Comment on 'Psychological distress in patients with cancer: is screening the effective solution?'
}

\begin{abstract}
J Dekker ${ }^{\star 1,2,3}$, A T F Beekman ${ }^{1,2}$, A D Boenink ${ }^{1}$, H Bomhof-Roordink ${ }^{1,2}$, A M Braamse ${ }^{1,2}$, E H Collette ${ }^{4}$, P C Huijgens ${ }^{3,5}, \mathrm{M} \mathrm{H}$ M van der Linden ${ }^{4}$, B van Meijel ${ }^{6}$, F J Snoek ${ }^{2,4}$, O Visser ${ }^{3,5}$ and H M Verheul ${ }^{3,7}$

${ }^{1}$ Department of Psychiatry, VU University Medical Center, Amsterdam, the Netherlands; ${ }^{2} E M G O$ Institute for Health and Care Research, Amsterdam, the Netherlands; ${ }^{3} \mathrm{VUmc}$ Cancer Center Amsterdam, Amsterdam, the Netherlands; ${ }^{4}$ Department of Medical Oncology, VU University Medical Center, Amsterdam, the Netherlands; ${ }^{5}$ Department of Medical Psychology, VU University Medical Center, Amsterdam, the Netherlands; ${ }^{6}$ Department of Hematology, VU University Medical Center, Amsterdam, the Netherlands and ${ }^{7}$ Inholland University of Applied Sciences, Research Group Mental Health Nursing; and Cluster Nursing, Amsterdam Parnassia Psychiatric Institute, The Hague
\end{abstract}

Sir,

Screening for psychological distress in patients with cancer is currently being debated in the British Journal of Cancer. Screening has been recommended, as elevated levels of distress have been consistently observed and clinicians tend to overlook the need of psychological support (Carlson et al, 2012; Carlson et al, 2013; National Comprehensive Cancer Network, 2013). On the other hand, it has been argued that screening should not be implemented, as the true benefit of screening and subsequent treatment of psychological distress is far from being definitively proven (Coyne, 2013).

Recent findings on human resilience in the face of potentially traumatic events (PTEs) provide a new perspective on detecting and treating psychological distress in patients with cancer. Humans show strong resilience in the face of potentially traumatic events, such as cancer diagnosis and treatment (Bonanno et al, 2011). This observation leads us to propose two alternative approaches towards detecting and treating psychological distress in patients with cancer: 'screening for psychological distress' and 'supporting resilience and case finding'.

\section{PREVALENCE OF PSYCHOLOGICAL DISTRESS}

A quantitative meta-analysis found that mood disorders were present in $38.2 \%$ (95\% CI: $28.4-48.6 \%$ ) of patients in oncology and haematology (Mitchell et al, 2011a). This review points to a high level of mood disorders in oncology and haematology. At the same time, this review shows that no signs of mood disorders were found in $100-38.2=61.8 \%$ of patients. Despite life-threatening disease and frequently intensive treatment, the majority of patients are remarkably resilient. They may have a hard time in coping with these events, they may be in need of support - they do not show signs of mood disorders, however.

Bonanno et al (2011) reviewed literature on individual differences in reaction to PTEs, such as violence, natural disaster, or a life-threatening diagnosis. They identified four prototypical trajectories (see Figure 1): 'Resilience is characterized by transient symptoms, minimal impairment, and a relatively stable trajectory of healthy functioning even soon after the PTE; recovery is distinguished from resilience by elevated symptoms and some functional impairment after the PTE followed by a gradual return to normal levels of functioning; chronic distress is characterized by a sharp elevation in symptoms and in functional impairment that may persist for years after the PTE; finally, delayed distress is characterized by moderate to elevated symptoms soon after the PTE and a gradual worsening across time' ( $\mathrm{p}$ 514-515). Resilience is the most frequent response to PTEs (Bonanno et al, 2011). This applies to patients with cancer as well: resilience was the most frequent trajectory in breast cancer surgery survivors and in patients with colorectal cancer, occurring in $66 \%$ and $67 \%$ of patients, respectively (Hou et al, 2010; Lam et al, 2010).

\section{SCREENING FOR PSYCHOLOGICAL DISTRESS}

The immediate implication of these observations is that it is not easy to demonstrate the effectiveness of screening. With resilience occurring so frequently, screening necessarily aims at a minority of patients: in a 


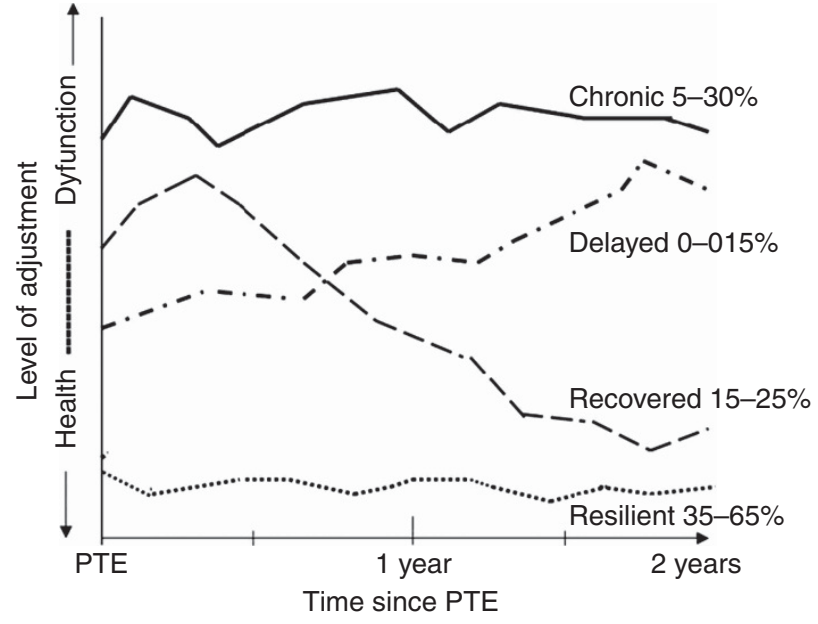

Figure 1. Four prototypical trajectories of adjustment after potentially traumatic events (PTEs). Reproduced from Bonanno et al (2011) by kind permission of Annual Reviews.

recent trial on screening, a referral for treatment was given to $19.6 \%$ and $26.3 \%$ of the patients with lung and breast cancer, respectively (Carlson et al, 2010). Even if treatment would be highly effective, screening and subsequent treatment has limited impact on the entire sample, as the majority of the patients is not distressed nor treated.

It can be argued that cancer care needs to be highly organised in order to make screening effective. Targeted selection, enhanced care, and stepped care seem essential organisational requirements. Targeted selection (or triage) involves administering and scoring of the screening instrument by someone other than the clinician most frequently a nurse; those with high scores are offered a short interview to explore the need for treatment (Gilbody et al, 2005; Carlson et al, 2010). Enhanced care involves training of clinicians and nursing staff, participation of nursing staff or a specialised mental health provider, and several follow-up contacts. (O'Connor et al, 2009; Gilbody and Beck, 2010; Beekman et al, 2013). Stepped care involves applying both nurse-led interventions and specialised mental health interventions. Psychologically trained nurses seem to be highly qualified to deliver first-line psychological interventions (Galway et al, 2012). Because of their biomedical training, nurses can address psychological issues in the context of somatic care, which results in relatively effective first-line interventions (Galway et al, 2012). If the nurse-led psychological intervention is not sufficiently effective, mental health experts (psychologist, psychiatrist) may offer specialised care.

\section{SUPPORTING RESILIENCE AND CASE FINDING}

Bonanno et al (2011) identified a number of predictors of resilience, including personality (e.g., capacity for positive emotions), resources (emotional and social support), and demographics (male gender, older age, and greater education). These predictors of resilience correspond remarkably well to predictors of quality of life in patients with cancer: a systematic review on patients with haematological malignancies receiving stem cell transplantations showed that poor quality of life was predicted by pre-transplant psychological distress, receiving less social support, female gender, younger age, graft- $v s$-host disease, and specific conditioning regimens (Braamse et al, 2012).

These findings open the possibility of an alternative to screening for psychological distress. Because approximately two-thirds of patients with cancer are likely to be resilient (Hou et al, 2010; Lam et al. 2010; Mitchell et al, 2011a), the logical approach would be to support resilience. Resilience may not come automatically dealing with a life-threatening disease and intensive treatment requires a huge personal effort. Patients may need emotional support, provided by family and friends, as well as clinicians and nurses during routine care at the hospital (Galway et al, 2012).

Patients less likely to be resilient can be identified using empirically derived predictors of poor resilience ('yellow flags'). We suggest that a set of factors indicating increased risk of poor resilience can be developed using simple indicators of emotional or social support (e.g., absence of informal care provided by family members or friends), demographics (e.g., younger age), and specific characteristics of disease and treatment (e.g., graft- $v s$-host disease). Information on yellow flags is usually available in the medical file and does not require extensive testing.

In patients identified as being less likely to show resilience, case finding could be instituted. Case finding is to be distinguished from screening, both conceptually and operationally: '... screening is the application of a diagnostic test or clinical assessment in order to optimally rule-out those without the disorder with minimal false negatives (missed cases). Screening is often performed in a large population as the first of several diagnostic tests. (...) ... Case finding $\langle i s\rangle \ldots$ the application of a diagnostic test or clinical assessment in order to optimally identify those with the disorder with minimal false positives (...). Case finding is often performed in a selected population at high risk for the condition' ( $\mathrm{p}$ 150) (Mitchell et al, 2011b; Mitchell et al, 2012). We suggest to rely on case finding for the selected population of patients with yellow flags, indicating increased risk of poor resilience. If case finding shows the presence of psychological distress, the patient's need for treatment should be discussed; and nurse-led interventions as well as specialised treatment by a mental health expert could be offered, similar to the approach based on screening.

In conclusion, research on resilience provides a new perspective, which may help to solve the debate on detecting and treating psychological distress in patients with cancer. Because the majority of patients with cancer is resilient and may not need psychological treatment, screening is likely to be effective only if screening and treatment are highly organised. Alternatively, one could rely on supporting resilience; patients in need of psychological treatment can be identified based on an empirically derived set of risk factors (yellow flags) and case finding.

\section{ACKNOWLEDGEMENTS}

Preparation of this work was partially supported by a grant from Alpe d'Huzes/ Dutch Cancer Society (VU 2011-5279).

\section{REFERENCES}

Beekman AT, van der Feltz-Cornelis C, van Marwijk HW (2013) Enhanced care for depression. Curr Opin Psychiatry 26: 7-12.

Bonanno GA, Westphal M, Mancini AD (2011) Resilience to loss and potential trauma. Annu Rev Clin Psychol 7: 511-535.

Braamse AM, Gerrits MM, van MB, Visser O, van OP, Boenink AD, Cuijpers P, Huijgens PC, Beekman AT, Dekker J (2012) Predictors of health-related quality of life in patients treated with auto- and allo-SCT for hematological malignancies. Bone Marrow Transplant 47: 757-769.

Carlson LE, Groff SL, Maciejewski O, Bultz BD (2010) Screening for distress in lung and breast cancer outpatients: a randomized controlled trial. J Clin Oncol 28: 4884-4891.

Carlson LE, Waller A, Groff SL, Zhong L, Bultz BD (2012) Online screening for distress, the 6th vital sign, in newly diagnosed oncology outpatients: randomised controlled trial of computerised vs personalised triage. $\mathrm{Br} \mathrm{J}$ Cancer 107: 617-625.

Carlson LE, Waller A, Groff SL, Zhong L, Bultz BD (2013) Reply: benefits of screening cancer patients for distress still not demonstrated. Br J Cancer 108: $738-739$. 
Coyne JC (2013) Benefits of screening cancer patients for distress still not demonstrated. Br J Cancer 108: 736-737.

Galway K, Black A, Cantwell M, Cardwell CR, Mills M, Donnelly M (2012) Psychosocial interventions to improve quality of life and emotional wellbeing for recently diagnosed cancer patients. Cochrane Database Syst Rev 11: CD007064.

Gilbody S, Beck D (2010) Implementing screening as part of enhanced care: screening alone is not enough. In Screening for Depression in Clinical Practice: An Evidence Based Guide, Mitchell AJ, Coyne JC (eds) pp 123-141. Oxford University Press: Oxford.

Gilbody S, House AO, Sheldon TA (2005) Screening and case finding instruments for depression. Cochrane Database Syst Rev 19: CD002792.

Hou WK, Law CC, Yin J, Fu YT (2010) Resource loss, resource gain, and psychological resilience and dysfunction following cancer diagnosis: a growth mixture modeling approach. Health Psychol 29: 484-495.

Lam WW, Bonanno GA, Mancini AD, Ho S, Chan M, Hung WK, Or A, Fielding R (2010) Trajectories of psychological distress among Chinese women diagnosed with breast cancer. Psychooncology 19: 1044-1051.
Mitchell AJ, Chan M, Bhatti H, Halton M, Grassi L, Johansen C, Meader N (2011a) Prevalence of depression, anxiety, and adjustment disorder in oncological, haematological, and palliative-care settings: a meta-analysis of 94 interview-based studies. Lancet Oncol 12: 160-174.

Mitchell AJ, Hussain N, Grainger L, Symonds P (2011b) Identification of patient-reported distress by clinical nurse specialists in routine oncology practice: a multicentre UK study. Psychooncology 20: 1076-1083.

Mitchell AJ, Meader N, Davies E, Clover K, Carter GL, Loscalzo MJ, Linden W, Grassi L, Johansen C, Carlson LE, Zabora J (2012) Meta-analysis of screening and case finding tools for depression in cancer: evidence based recommendations for clinical practice on behalf of the Depression in Cancer Care consensus group. J Affect Disord 140: 149-160.

National Comprehensive Cancer Network (2013) Guideline Distress Management Version 2.2013. NCCN. Ref type: Electronic citation.

O'Connor EA, Whitlock EP, Beil TL, Gaynes BN (2009) Screening for depression in adult patients in primary care settings: a systematic evidence review. Ann Intern Med 151: 793-803. 